\title{
Numerical Simulation of Projectile Oblique Impact on Microspacecraft Structure
}

\author{
Zhiyuan Zhang, Runqiang Chi, Baojun Pang, and Gongshun Guan \\ Hypervelocity Impact Research Center, Harbin Institute of Technology, Harbin 150080, China \\ Correspondence should be addressed to Runqiang Chi; chirq@hit.edu.cn
}

Received 25 October 2016; Revised 25 January 2017; Accepted 20 February 2017; Published 16 April 2017

Academic Editor: Enrico C. Lorenzini

Copyright (c) 2017 Zhiyuan Zhang et al. This is an open access article distributed under the Creative Commons Attribution License, which permits unrestricted use, distribution, and reproduction in any medium, provided the original work is properly cited.

\begin{abstract}
In the present study, the microspacecraft bulkhead was reduced to the double honeycomb panel, and the projectile oblique hypervelocity impact on the double honeycomb panel was simulated. The distribution of the debris cloud and the damage of a honeycomb sandwich panel were investigated when the incident angles were set to be $60^{\circ}, 45^{\circ}$, and $30^{\circ}$. The results showed that as incident angle decreased, the distribution of debris cloud was increased gradually, while the maximum perforation size of the rear face sheet was firstly increased with the decrease of the incident angle and then decreased. On the other hand, the damage area and the damage degree of the front face sheet of the second honeycomb panel layer were increased with the decrease of the incident angle. Finally, the critical angle of front and rear face sheets of the honeycomb sandwich panel was obtained under oblique hypervelocity impact.
\end{abstract}

\section{Introduction}

A large amount of the space debris has been accumulated as the increase of the human space activities within the scope of the near-earth space, which posed a serious threat to the safe operation of the spacecraft in orbit $[1,2]$. Honeycomb sandwich panel is commonly used as a kind of structure material of the spacecraft bulkhead which is made up of panels and honeycomb core sticking together $[3,4]$. The space debris can easily penetrate honeycomb sandwich panel with an average speed of $10 \mathrm{~km} / \mathrm{s}$ in the earth orbit $[1,3]$. As for the spacecraft bulkhead, the honeycomb sandwich panel will be firstly suffered the impact of the space debris $[5,6]$.

A lot of researches have analyzed the damage characteristics induced by the impact of the space debris on the monolayer honeycomb sandwich panel [7] by experiments and numerical simulations by considering the effects of size $[8]$, the impact velocity $[8,9]$, the materials $[10]$, and the collision limit [11]. But the hypervelocity impact on double honeycomb sandwich panels which is different from two layer honeycomb sandwich panels being bonded together [5] has not yet been reported. One side of the spacecraft bulkhead will be penetrated; then the inside the equipment and the other side of the bulkhead can also be damaged due to the high speed and the large kinetic energy of the space debris. The study of the effects of the high speed impact on the front and rear bulkheads is necessary.

In view of the space debris impact on both sides of the spacecraft bulkhead, the spacecraft was simplified to the structure of double honeycomb sandwich panel, which used to study the breaking condition of the projectile and the damage of the honeycomb sandwich panel under the oblique impact. Finally, the critical incident angle of the single honeycomb sandwich panel is calculated in this paper.

\section{Model of Simulation}

Figure 1 shows a double honeycomb sandwich panel structure of the simplified model of the spacecraft. The distance between two layers of the honeycomb sandwich plates is $500 \mathrm{~mm}$ and the space debris is simplified as spherical aluminum alloy projectiles. Smoothed Particle Hydrodynamics $(\mathrm{SPH})$ is used as aluminum alloy projectile model which can solve the problem that the mesh deformation of projectile is too large. Finite element method (FE) is used in the honeycomb sandwich panel model and the shell element is used for the honeycomb core. Particle size of SPH is set to be $0.2 \mathrm{~mm}$. Grid size near impact point and outside 
TABLE 1: Description of the simulation model.

\begin{tabular}{lccccc}
\hline & Material & Dimension $/ \mathrm{mm}$ & EOS & Strength & Failure \\
\hline Projectile & Al 2017 & Sphere $/ D=5$ & Shock & Johnson-Cook & Principal stress \\
Face sheet & Al 5A06 & $h=0.8$ & Shock & Johnson-Cook & Plastic strain \\
Honeycomb core & Al 5A06 & $L=4, H=20, t=0.025$ & Linear & Johnson-Cook & Plastic strain \\
\hline
\end{tabular}

TABLE 2: Parameters of materials [12].

\begin{tabular}{lccccccc}
\hline & $A / \mathrm{MPa}$ & $B / \mathrm{MPa}$ & $n$ & $C$ & $m$ & $T_{m} / \mathrm{K}$ & $\mathrm{Density} / \mathrm{g} \cdot \mathrm{cm}^{-3}$ \\
\hline Al 5A06 & 235.4 & 622.3 & 0.58 & 0.0174 & 1.05 & 853 & 2.64 \\
Al 2017 & 249.9 & 426.0 & 0.34 & 0.015 & 1.0 & 775 & 2.79 \\
\hline
\end{tabular}

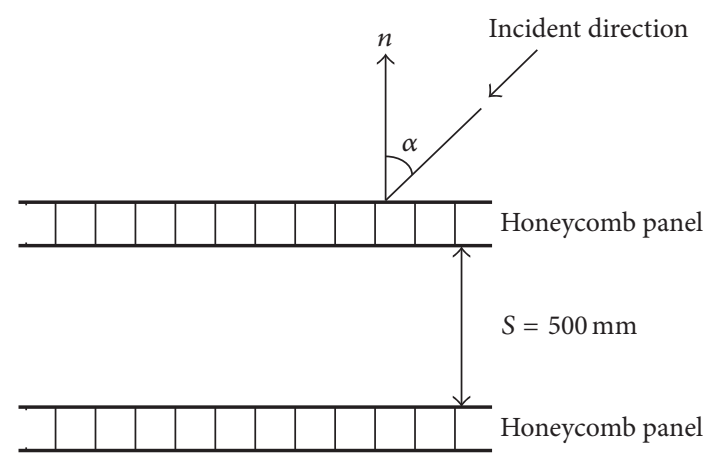

Figure 1: Double honeycomb sandwich panel structure diagram.

are set to be $0.3 \mathrm{~mm}$ and $0.8 \mathrm{~mm}$. Each honeycomb core is equal to the grid on the panel correspondingly. By using the model in Figure 2, the nodes of honeycomb core and panel grids are processed. The process of the projectile impact on honeycomb sandwich panel at a high speed was simulated using AUTODYN 15.0 and the simulation parameters were shown in Table 1. Geometric strain was used as the erosion model, and the value was set 2 . In this model, $D$ is the projectile diameter, $h$ is thickness of honeycomb sandwich panel, $L$ is a side length of hexagonal honeycomb core, $H$ is height of the honeycomb core, and $t$ is thickness of cellular wall. The model parameters of the honeycomb sandwich panel in this paper were based on data from microspacecraft project, which can apply to communication, ground remote sensing, the interplanetary exploration, scientific research, and so forth.

Johnson-Cook model can describe the nonlinear process of the high speed impact, which can be written as

$$
\sigma=\left(A+B \varepsilon^{n}\right)\left(1+C \ln \dot{\varepsilon}^{*}\right)\left(1-T^{* m}\right)
$$

in which $A, B, n, C, m$ are constants related to materials, $\dot{\varepsilon}^{*}$ is the ratio of reference strain rate and strain rate, $T^{*}=(T-$ $\left.T_{r}\right) /\left(T_{m}-T_{r}\right), T_{r}$ is room temperature $(300 \mathrm{~K})$, and $T_{m}$ is the melting point of materials. Material parameters of numerical simulation are shown in Table 2.

We have validated the accuracy of the simulation model by experiment as shown in Figure 3 [13] and Table 2. The parameters of the projectile and the honeycomb sandwich panel were shown in Table 1 . The impact velocity of projectile was $1.915 \mathrm{~km} / \mathrm{s}$. The perforation diameter of rear face sheet

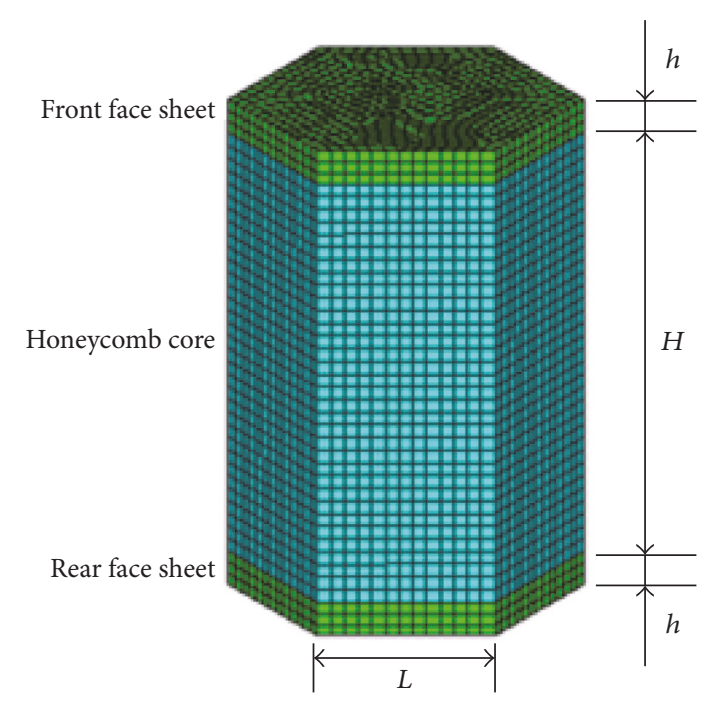

FIgURe 2: Honeycomb panel model.

is about $14 \mathrm{~mm}$ in both experimental and simulation results. The others experiments were shown in Table 3 . The experimental results coincide with the simulation results, which verified the correctness of the simulation model.

\section{Projectile Oblique Impact on Structure of Double Honeycomb Sandwich Panel}

3.1. The Configuration of Debris Cloud. Half symmetry model was adopted, including 145164 solid elements, 239056 shell elements, and $4072 \mathrm{SPH}$ particles. The projectile velocity is set to be $3.07 \mathrm{~km} / \mathrm{s} .3 \mathrm{~km} / \mathrm{s}$ (or lower) was studied because it is in the extent of speed of orbital debris, and less than or equal to $3 \mathrm{~km} / \mathrm{s}$ is an important part of the ballistic limit. Every $\mathrm{SPH}$ particle contains various kinds of physical quantities, such as mass and speed, and so forth. The distribution of debris cloud can be described by the analysis of the projectile mass fraction of debris cloud. The distribution of debris cloud after the projectile impacted the first layer of honeycomb sandwich panel was shown in Figure 4. In Figure 4(a), the debris cloud distribution area was divided into three regions. Region I was the part above the front face sheet of honeycomb sandwich panel, which was the backwash debris cloud. Region II was the interior of the honeycomb sandwich panel, which was 
TABLE 3: Experiment and simulation results of rear face sheet of honeycomb sandwich panel.

\begin{tabular}{lcccrr}
\hline & $\begin{array}{c}\text { Projectile } \\
\text { diameter/mm }\end{array}$ & Velocity/km·s $\mathrm{s}^{-1}$ & $\begin{array}{c}\text { Experiment } \\
\text { result/mm }\end{array}$ & $\begin{array}{c}\text { Simulation } \\
\text { result/mm }\end{array}$ \\
\hline Test 1 & 5 & 1.915 & 14.1 & 15.3 & $7.8 \%$ \\
Test 2 & 8 & 1.7 & 22.5 & 24.6 & $8.5 \%$ \\
Test 3 & 5 & 3.065 & 25.4 & 23.9 & $5.9 \%$ \\
\hline
\end{tabular}

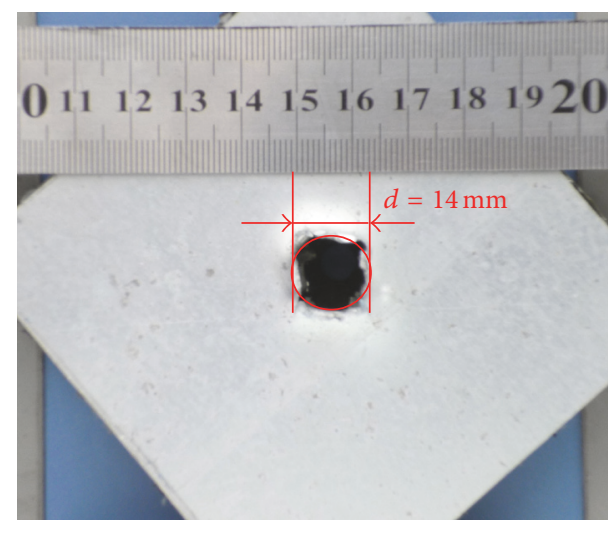

(a) Experimental result

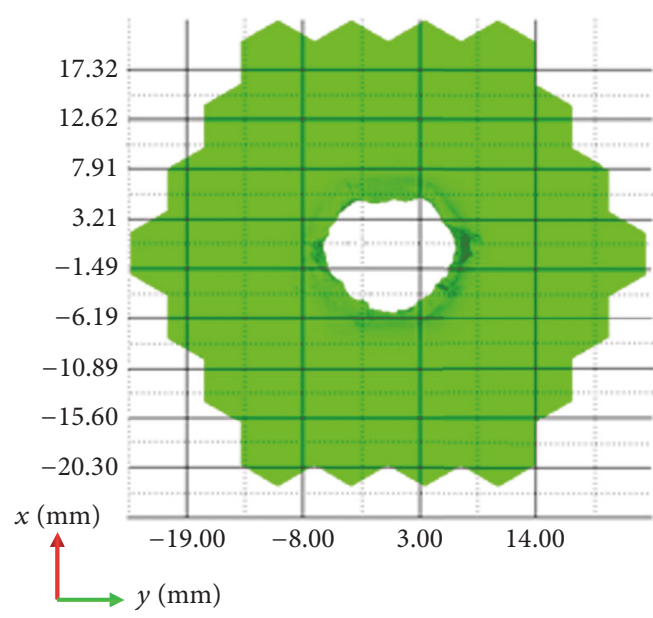

(b) Simulation result

FIgURE 3: Experiment and simulation results [12].

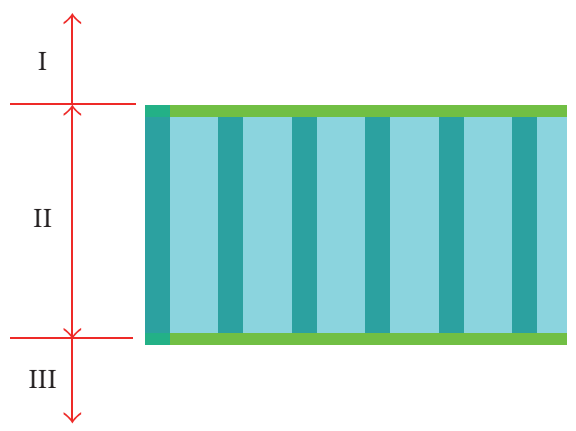

(a) The division of debris cloud distribution area

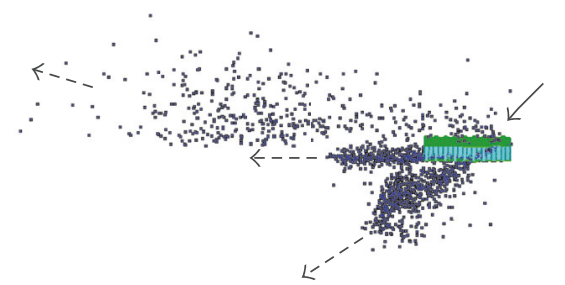

$\longleftarrow$ Incident direction of the projectile $\leftarrow--$ Sputtering direction of debris cloud

(b) $\alpha=60^{\circ}$

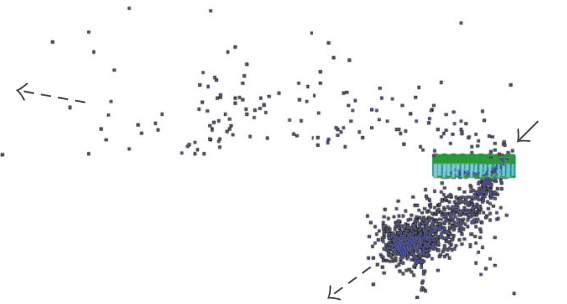

$\longleftarrow$ Incident direction of the projectile $\leftarrow--$ Sputtering direction of debris cloud

(c) $\alpha=45^{\circ}$

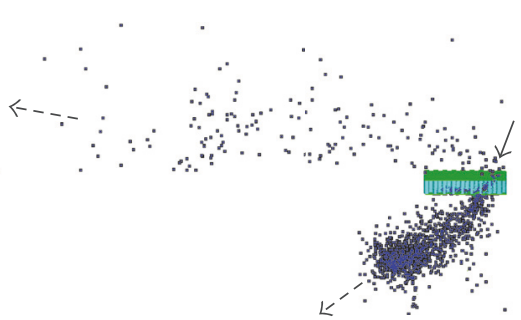

$\longleftarrow$ Incident direction of the projectile $\leftarrow--$ Sputtering direction of debris cloud

(d) $\alpha=30^{\circ}$

FIgURE 4: The distribution of the debris cloud in $9.5 E-002 \mathrm{~ms}$. 


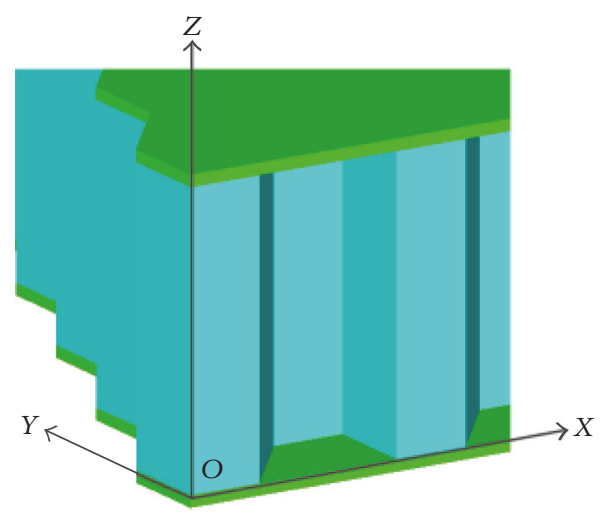

(a) Coordinate system

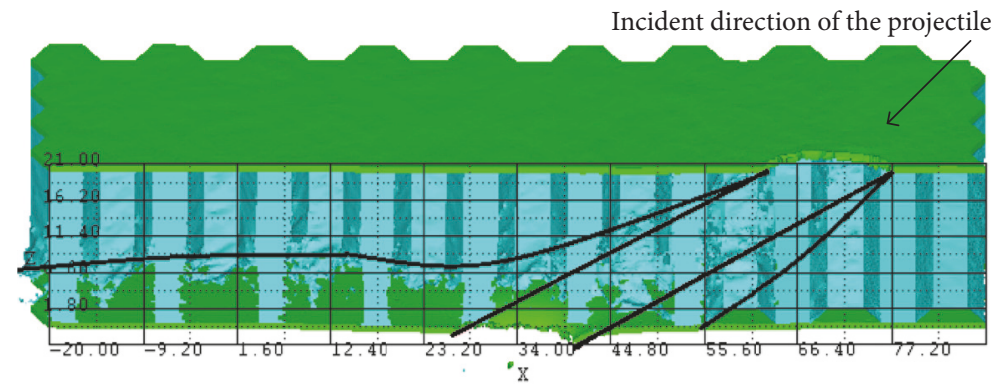

(b) $\alpha=60^{\circ}$

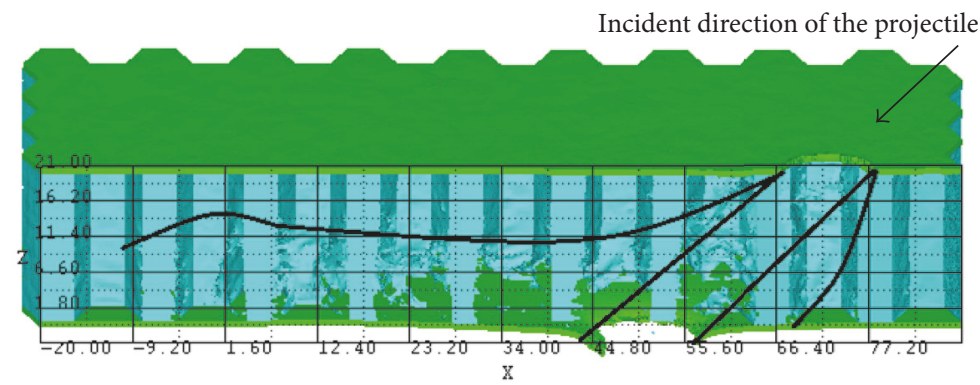

(c) $\alpha=45^{\circ}$

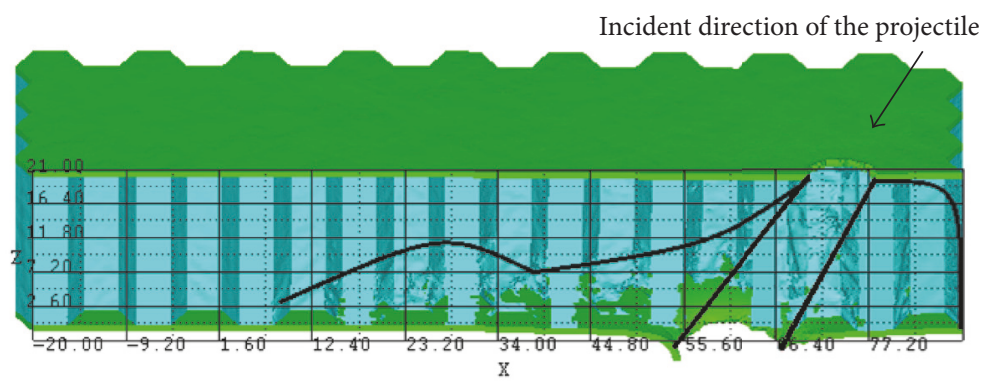

(d) $\alpha=30^{\circ}$

FIGURE 5: Damage of the first layer of honeycomb sandwich panel.

made of the radial motion parts of projectile debris cloud and the backwash debris cloud which formed due to influence of the rear face sheet. Region III was the part below rear face sheet, which was the debris cloud part which go through the rear face sheet.

Figures 4(b), 4(c), and 4(d) show the distribution of debris cloud of projectile when the impact angle was $60^{\circ}$, $45^{\circ}$, and $30^{\circ}$, respectively. The debris cloud distribution was obviously influenced by the impact angles. In order to analyze the distribution of debris cloud accurately, the projectile mass fraction of the debris cloud in different debris cloud distribution areas was obtained as shown in Table 4. From Figure 4 and Table 4, it can be seen that Region III increased gradually with the decrease of the impact angle. The projectile debris cloud was mainly distributed in Region III when the impact angle was $30^{\circ}$, and the main movement direction of the debris cloud was in the vertical direction.
TABLE 4: Projectile mass fraction of debris cloud.

\begin{tabular}{lccc}
\hline Incident angle & Region I & Region II & Region III \\
\hline $60^{\circ}$ & $18.33 \%$ & $33.33 \%$ & $48.33 \%$ \\
$45^{\circ}$ & $4.76 \%$ & $23.67 \%$ & $71.67 \%$ \\
$30^{\circ}$ & $1.67 \%$ & $18.33 \%$ & $80.00 \%$ \\
\hline
\end{tabular}

3.2. Damage of the First Layer of Double Honeycomb Sandwich Panel. Damage of the panel and honeycomb core after the projectile impact the first layer of double honeycomb sandwich panel was formed as shown in Figure 5. The debris cloud of projectile enters the honeycomb sandwich panel inside can be divided into two parts, one was the "main part" which was between the two straight lines as shown in Figure 5, and the other part was the "secondary part" which was between the two curves except "main part"; this part 


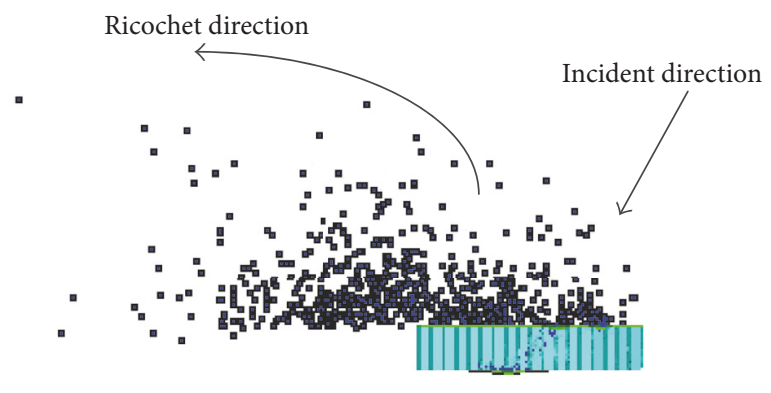

(a) The configuration of debris cloud

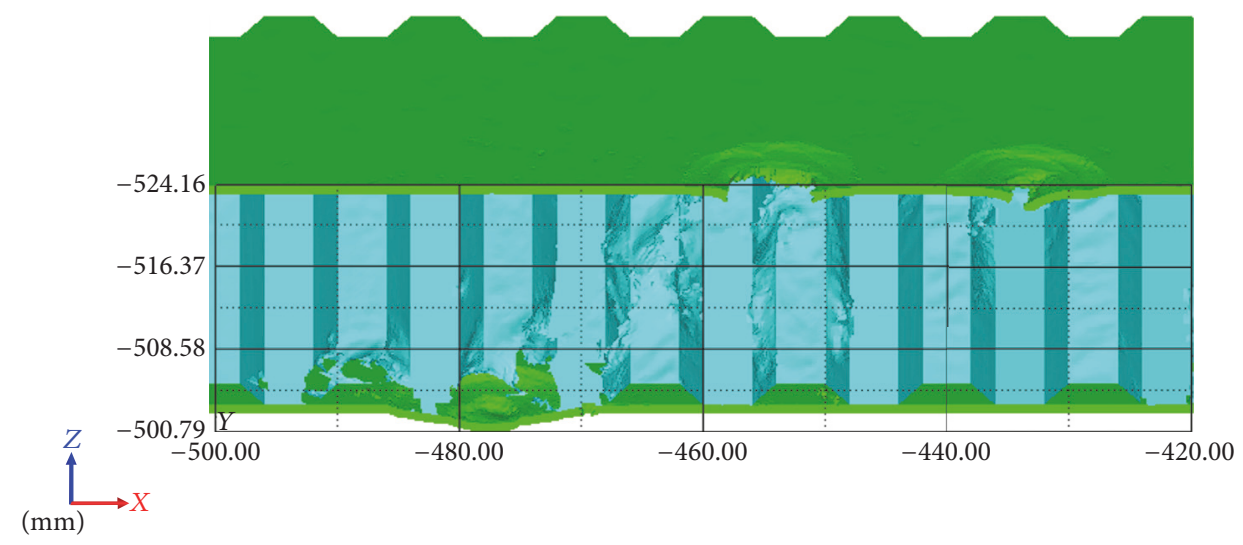

(b) Damage of the second layer of double honeycomb sandwich panel

FIgURE 6: Second layer of double honeycomb sandwich panels (incidence angle is $45^{\circ}$ ).

of debris cloud did not have enough kinetic energy to get through the panel but only expanded inside the honeycomb core after the backwash and then penetrated into the cellular wall. Since penetrating the cellular wall will consume the kinetic energy of debris cloud, the energy of the debris cloud decreased so that it cannot penetrate into the honeycomb core again after penetrating a certain number of the cellular walls.

A coordinate system was given to describe the damage degree of honeycomb panel (Figure 5(a)), and the coordinate origin can move along $x$-axis. With the decrease of the projectile impact angle, the projectile velocity along $x$-axis was reduced accordingly. The projectile mass fraction of inflation debris cloud along $x$-axis was also reduced. As a result, damage area of the honeycomb core caused by debris cloud was decreased. It can be seen from Figure 6 that the expansion scope of debris cloud was reduced with the decrease of the impact angle. When the impact angle was $30^{\circ}$ (Figure $5(\mathrm{~d})$ ), the damage range of the honeycomb core was reduced significantly compared with $60^{\circ}$ (Figure $5(\mathrm{~b})$ ) and $45^{\circ}$ (Figure 5(c)). Thus, there are obvious relationships between the damage of honeycomb sandwich panel and the impact angle.

Different impact angles lead to various perforation shape and size of rear face sheet as shown in Table 5. It can be found that the perforation shape can be described by an ellipse approximately. The perforation shape was more likely an oval shape when the impact angle was $60^{\circ}$ compared with that of $45^{\circ}$ and $30^{\circ}$; this was induced by the degeneration of elliptic equations.
As shown in Table 5, the largest perforation size of the front face sheet reduces with the decrease of the impact angle. Meanwhile, the largest perforation size of the rear face sheet was increased firstly and then decreased. The largest perforation size of the front face sheet was slightly greater than the rear face sheet when the impact angle was $60^{\circ}$, but the former is significantly smaller than the latter when the impact angle was $45^{\circ}$ and $30^{\circ}$. This is because the velocity component of $z$-axis was larger when the impact angle was smaller and more debris cloud particles impact the rear face sheet, resulting in the greater damage in the rear face sheet.

\subsection{Damage of the Second Layer of Double Honeycomb} Sandwich Panel. For the double honeycomb sandwich panel structure, the projectile was broken after it penetrated the first layer of the honeycomb sandwich panel, and then the projectile continued to move in the form of debris cloud until it impacted the second layer of honeycomb sandwich panel. The shape of debris cloud was related to the incident angle of the projectile, including the projectile broken partly or completely. The damage degree of rear face sheet was associated with the shape of projectile debris cloud. Because of the size and velocity, the nonsignificant fragment of front face sheet of the first layer of honeycomb sandwich panel was observed, so we ignored the effect of fragment of face sheet on damage in this paper.

The debris cloud, which formed after the projectile penetrated into the first layer of the honeycomb sandwich panel, impacted the second layer of honeycomb sandwich panel, 
TABLE 5: The perforation shape of panel.

Front face sheet

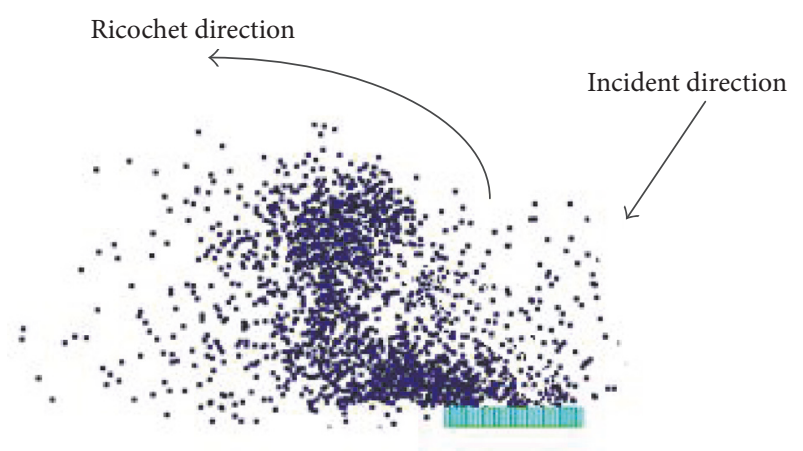

(a) The configuration of debris cloud

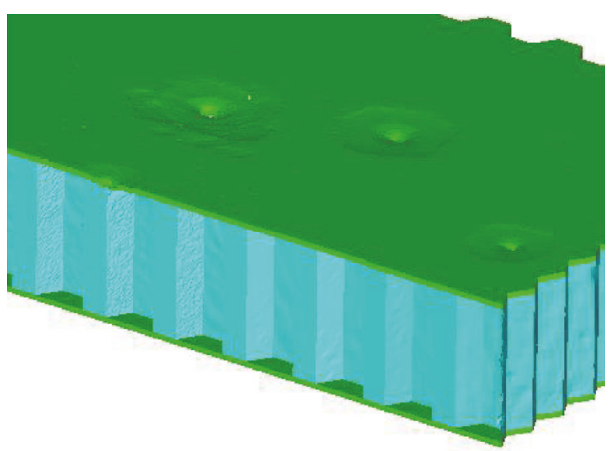

(b) Damage of the second layer of honeycomb sandwich panel

FIGURE 7: Second layer of honeycomb sandwich panels (incidence angle is $30^{\circ}$ ).

where distance to the first layer is $500 \mathrm{~mm}$. The projectile mass fraction of debris cloud which penetrated the first layer of the honeycomb sandwich panel was not much and broken fully (Figure 4(b)); the kinetic energy of debris cloud was too small to induce obvious damage on the honeycomb sandwich panel plate when impact angle was $60^{\circ}$. Therefore, damage of the second layer of honeycomb sandwich panel was studied only under the case of the impact angles of $45^{\circ}$ and $30^{\circ}$ in this paper, as shown in Figures 6 and 7.

Figure 4(c) shows the projectile debris cloud form on the situation that the projectile has penetrated the first layer of honeycomb sandwich panel but did not impact the second layer when the impact angle was $45^{\circ}$. Big pieces did not break in the front of debris cloud, which are the main factors that lead to the perforation on front face sheet and then impacted the second layer of double honeycomb sandwich panel. Most of the scattered debris cloud cannot penetrate the front face sheet of second layer of double honeycomb sandwich panel, but splash after impacted the front face sheet as shown in Figure 6(a). Figure 6(b) shows the damage of the second layer of honeycomb sandwich panel. According to the two perforations in front face sheet, we can know that there were two pieces of debris that impacted the front face sheet and formed punches and smaller piece of debris fully broken after getting through the front face sheet so that it cannot cause damage to the rear face sheet again. Large pieces of debris impact the rear face sheet after penetrating the front face sheet. The kinetic energy of debris cloud was too small to penetrate the back panel at that moment and only craters in the rear face sheet were formed.

Distribution and damage of debris cloud after the projectile impact the second layer of honeycomb sandwich panel of double when impact angle was $30^{\circ}$ as shown in Figure 7. There are three obvious perforations on the rear face sheet. The projectile was further broken when debris cloud penetrated the second layer of honeycomb sandwich panel so that the rear face sheet without obvious damage due to the velocity component of $z$-axis at the impact angle of $30^{\circ}$ is larger than $45^{\circ}$.

According to the analysis of the damage of projectile impact double honeycomb sandwich panel, it can be found that the impact damage was closely related to the oblique impact angle. The projectile broken degree, the debris cloud distribution, and the damage on each layer of honeycomb sandwich panel were different under different impact angles. Ruining of the first layer of honeycomb sandwich panel was the most serious when projectile oblique impact double honeycomb sandwich panel structure. Debris cloud forms 


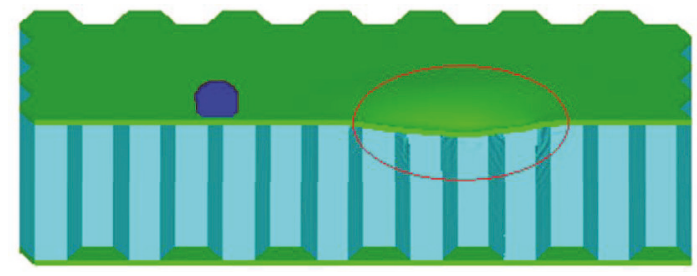

(a) Impact angle is $87^{\circ}$

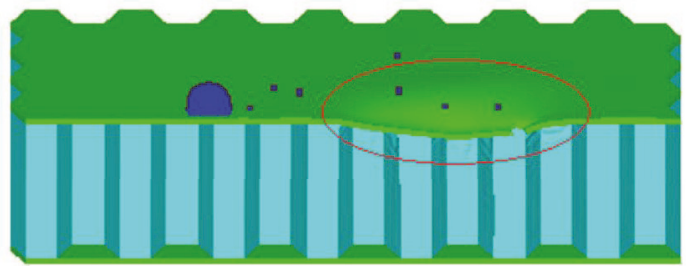

(b) Impact angle is $85^{\circ}$

FIgURE 8: Critical penetration of the front face sheet.

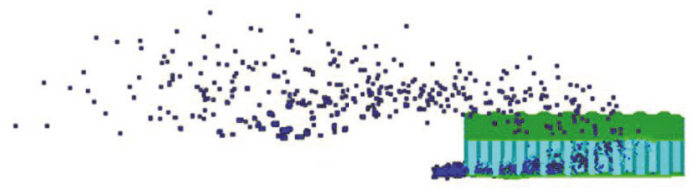

(a) Impact angle is $72^{\circ}$

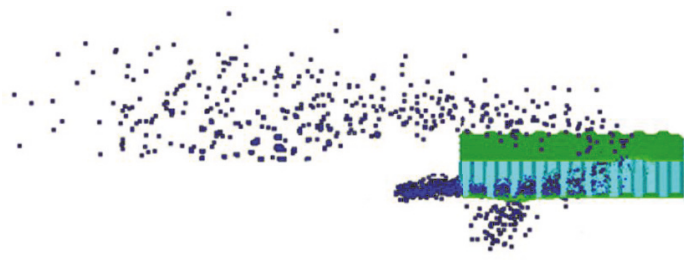

(b) Impact angle is $70^{\circ}$

FIGURE 9: Critical penetration of the rear face sheet.

were uncommon after the projectile penetrated the first layer of honeycomb sandwich panel at different impact angles, resulting the different damage shapes on the second layer of honeycomb sandwich panel. Incident angle of space debris impact the spacecraft is also different because the space debris and spacecraft orbit were different in the real space environment. It is terrible that the internal equipment of spacecraft and the other side of the bulkhead will be damaged if the space debris penetrated one side of the bulkhead. Critical penetration angle of the projectile oblique impact single honeycomb sandwich panel was studied in this paper based on using single honeycomb sandwich panel to simulate one side of the spacecraft bulkhead.

\section{Critical Penetration Angle of Projectile Oblique Impact on Honeycomb Sandwich Panel}

4.1. Critical Penetration Angle of the Front Face Sheet. The projectile penetrated first layer honeycomb sandwich panel would lead to more or less damage on the second layer honeycomb sandwich panel according to the study of the double honeycomb sandwich panel structure. On the other hand, critical perforation angle of single layer honeycomb sandwich panel is a part of a double honeycomb sandwich panel structure, so the critical penetration angle of projectile oblique impact on honeycomb sandwich panel was studied firstly.

The critical penetration is infinitely close to penetration and unpenetration. The critical penetration angle of the front face sheet of a honeycomb sandwich panel was found through a lot of simulation tests based on dichotomy. Impact speed is $3 \mathrm{~km} / \mathrm{s}$, for example, to analyze the critical penetration angle of the front face sheet. The critical angle that the front face sheet cannot be penetrated was $87^{\circ}$ as shown in Figure 8(a).
The projectile was not broken, and only plastic deformation occurred at that time. Impact craters were formed on the front face sheet of the honeycomb sandwich panel, but it was unable to be punched. Critical penetration angle of the front face sheet was $85^{\circ}$ as shown in Figure 8(b). Local plastic deformation and broken of projectile occurred, and there were holes on the front face sheet at the same moment, so the critical penetration angle of the front face sheet was $86^{\circ}$.

It can be viewed according to Figure 9 that projectile was not broken when projectile angle was greater than the critical penetration angle of the front face sheet. Therefore, critical penetration angle of the front face sheet is also the critical value of projectile broken. Symbol $C$ in this formula represents the collection of projectiles, which were not broken (see (2)) and Symbol $\bar{C}$ represents the collection of projectiles, which were broken (see (3)), $\theta$ represents the projectile incident angle, and $\widehat{\theta}$ represents critical penetration angle of the front face sheet:

$$
\begin{aligned}
& C=\left\{\theta>\widehat{\theta} \mid 0^{\circ} \leq \theta \leq 90^{\circ}\right\} \\
& \bar{C}=\left\{\theta>\widehat{\theta} \mid 0^{\circ} \leq \theta \leq 90^{\circ}\right\} .
\end{aligned}
$$

4.2. Critical Penetration Angle of the Rear Face Sheet. The critical angle of the rear face sheet of honeycomb sandwich panel was $72^{\circ}$ at the impact speed of $3 \mathrm{~km} / \mathrm{s}$ as shown in Figures 9(a) and 10(a). Projectile was broken after impacting the front face sheet and part of the debris cloud backwash then moved along the horizontal direction. Meanwhile, another part of the debris cloud moves into the honeycomb core layer. The motion of the debris cloud lateral leaded to the rupture and the collapse of the honeycomb core layer.

The critical angle of the rear face sheet of honeycomb sandwich panel was $70^{\circ}$ as shown in Figures 9(b) and 10(b). Compared to the incidence angle of $72^{\circ}$, the number of SPH 
TABLE 6: Particle diameter at ballistic limit (the particle diameter of simulation was $3 \mathrm{~mm}$ ).

\begin{tabular}{lccccccc}
\hline Velocity $/ \mathrm{km} \cdot \mathrm{s}^{-1}$ & 1.8 & 2 & 2.2 & 2.4 & 2.6 & 2.8 & 3 \\
\hline Calculation results of $(4) / \mathrm{mm}$ & 3.53 & 3.36 & 3.34 & 3.22 & 3.07 & 2.92 & 2.79 \\
\hline
\end{tabular}

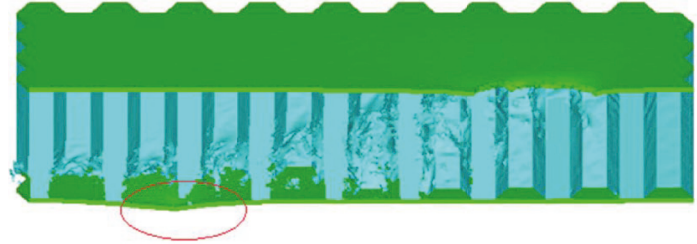

(a) Impact angle is $72^{\circ}$

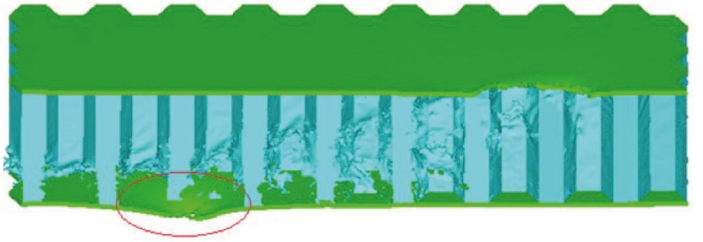

(b) Impact angle is $70^{\circ}$

FIGURE 10: Damage of rear face sheet with critical perforated.

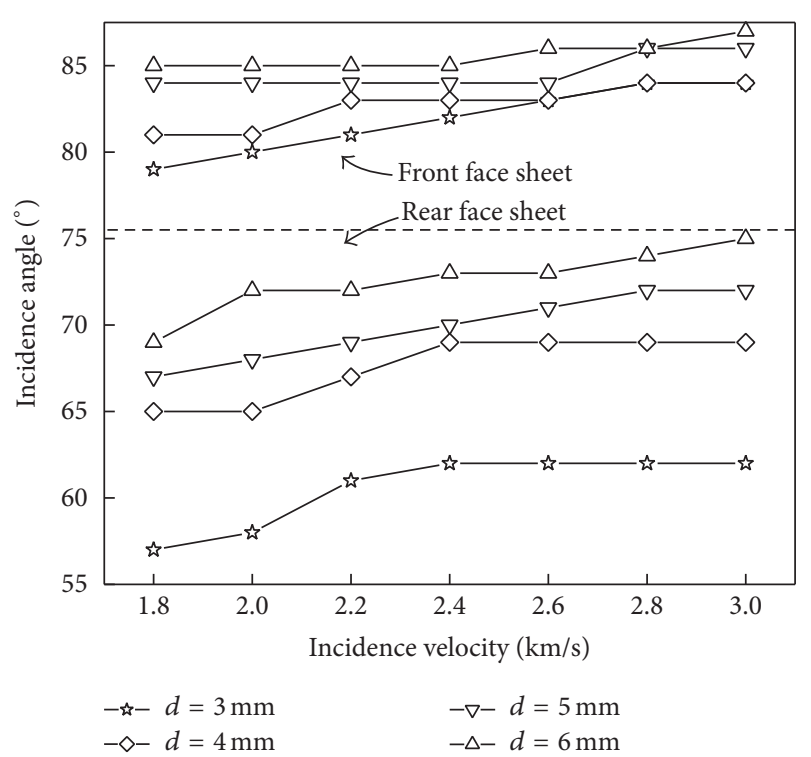

Figure 11: Critical perforation angles under various diameter and velocity of projectile.

particles that enter inside of honeycomb core and vertical component of the projectile velocity was increased. Damage on the rear face sheet was also increased so that the critical perforation at the incidence angle of $70^{\circ}$ occurred in rear face sheet. Therefore, the critical perforation angle of the rear face sheet was $71^{\circ}$.

The ability of projectile penetrating honeycomb sandwich panel is different when the impact speed of projectile or diameter is different. In order to study the critical perforation angle of front and rear face sheets of honeycomb sandwich panel at different impact speeds or diameters when oblique impacting, the impact test was simulated within the impacting speed range of $1.8 \sim 3.4 \mathrm{~km} / \mathrm{s}$ and the diameter is $3 \mathrm{~mm}$, $4 \mathrm{~mm}, 5 \mathrm{~mm}$, and $6 \mathrm{~mm}$ as shown in Figure 11.

Simulation results were compared with the ballistic limit of a sandwich panel in order to testify the accuracy of the simulation. Equation (4) is an example of the generic form of the ballistic limit of a sandwich panel [14]. Particle diameter at ballistic limit was calculated at a diameter of $3 \mathrm{~mm}$ as shown in Table 6. The simulation result showed good agreement with the calculation results.

$$
d_{c}\left(v_{n}\right)=\left[\frac{\left(t_{w} / K_{3 S}\right)(\tau / 40000)^{0.5}+t_{b}}{0.6 \cos \theta v_{n}^{0.677} \rho_{p}^{0.5}}\right]^{0.947}
$$

In (4), $d_{c}$ is a particle diameter at ballistic limit, $t_{w}$ is thickness of rear wall $(\mathrm{cm}), K_{3 S}$ is a baseline (1.4 [15]), $\tau$ is rear wall yield stress, $t_{b}$ is thickness of bumper, $\theta$ is incidence angle, $v_{n}$ is normal component of velocity, and $\rho_{p}$ is projectile density.

It can be seen that the critical perforation angle of front and rear face sheet increased slowly or are unchanged with the increase of impact velocity. The critical perforation angle of rear face sheet increased dramatically with the increase of diameter, especially the diameter from $3 \mathrm{~mm}$ to $4 \mathrm{~mm}$, but the front face sheet increased slowly.

\section{Conclusions}

The distribution of the debris cloud and the damage of double honeycomb sandwich panel were simulated under oblique hypervelocity impact. In addition, the critical penetration angle of front and rear face sheets of honeycomb sandwich panel was obtained. The conclusions are as follows.

Projectile mass fraction of debris cloud decreases with the decrease of the impact angle which back-splashed and stayed inside of the first layer of honeycomb sandwich panel and then across the honeycomb core. However, the damage of first layer of honeycomb sandwich panel increased gradually. That result from the biggest perforation size of the front face sheet decreased accordingly, and the biggest perforation size of the rear face sheet increased firstly but then decreased.

The projectile mass fraction of debris cloud which get through the first layer of honeycomb sandwich panel was too little to damage the second layer when the impact angle was larger, but it increased gradually with the decrease of impact angle so that the damage of the front face sheet of second layer honeycomb panel became more and more obvious, and the damage degree of the second layer was related to crushing degree of projectile after penetrated the first layer. The projectile will not break when projectile angle was greater than the critical perforation angle of the front face sheet, but 
it will break gradually when projectile angle is less than the critical perforation angle.

The critical perforation angle of front and rear face sheet increased slowly or is unchanged with the increase of impact velocity. The critical perforation angle of rear face sheet increased dramatically with the increase of diameter, especially the diameter from $3 \mathrm{~mm}$ to $4 \mathrm{~mm}$, but the front face sheet increased slowly.

For the research on the projectile oblique hypervelocity impact on double honeycomb panel, we only embarked on the study the distribution of projectile debris cloud and the level of damage of honeycomb panel under a velocity $(3.07 \mathrm{~km} / \mathrm{s})$ and three incidence angles $\left(60^{\circ}, 45^{\circ}\right.$, and $\left.30^{\circ}\right)$ of projectile. Therefore, future studies are worthy to be carried out to explore this subject further, such that a wide range of the speed conditions will be investigated, especially at higher velocity (about $10 \mathrm{~km} / \mathrm{s}$ ). On the other hand, different geometric models that will affect the fragmentation degree of projectile, which in turn will affect the damage degree of honeycomb panel, will also be studied. At last, we expect to obtain the critical penetration angle of projectile oblique impact on double honeycomb sandwich panel by more experiments.

\section{Conflicts of Interest}

The authors declare that they have no conflicts of interest.

\section{Acknowledgments}

This work was supported by the National Natural Science Foundation of China (11172083), the Aeronautical Science Foundation of China (2016ZD53036), and the Fundamental Research Funds for the Center Universities (HIT.NSRIF.2015029).

\section{References}

[1] J.-C. Liou and N. L. Johnson, "Risks in space from orbiting debris," Science, vol. 311, no. 5759, pp. 340-341, 2006.

[2] F. Zuiani and M. Vasile, "Preliminary design of debris removal missions by means of simplified models for low-thrust, manyrevolution transfers," International Journal of Aerospace Engineering, vol. 2012, Article ID 836250, 22 pages, 2012.

[3] S. Ryan, F. Schaefer, R. Destefanis, and M. Lambert, "A ballistic limit equation for hypervelocity impacts on composite honeycomb sandwich panel satellite structures," Advances in Space Research, vol. 41, no. 7, pp. 1152-1166, 2008.

[4] W. Schonberg, F. Schäfer, and R. Putzar, "Hypervelocity impact response of honeycomb sandwich panels," Acta Astronautica, vol. 66, no. 3-4, pp. 455-466, 2010.

[5] E. A. Taylor, J. P. Glanville, R. A. Clegg, and R. G. Turner, "Hypervelocity impact on spacecraft honeycomb: hydrocode simulation and damage laws," International Journal of Impact Engineering, vol. 29, no. 1, pp. 691-702, 2003.

[6] M. Higashide, N. Onose, and S. Hasegawa, "Sub-millimeter debris impact damage of unmanned spacecraft structure panel," Procedia Engineering, vol. 58, pp. 517-525, 2013.
[7] K. Nitta, M. Higashide, Y. Kitazawa, A. Tekaba, M. Katayama, and H. Matsumoto, "Response of a aluminum honeycomb subjected to hypervelocity impacts," Procedia Engineering, vol. 58, pp. 709-714, 2013.

[8] P. Kang, S.-K. Youn, and J. H. Lim, "Modification of the critical projectile diameter of honeycomb sandwich panel considering the channeling effect in hypervelocity impact," Aerospace Science and Technology, vol. 29, no. 1, pp. 413-425, 2013.

[9] S. Ryan, F. Schaefer, and W. Riedel, "Numerical simulation of hypervelocity impact on CFRP/Al HC SP spacecraft structures causing penetration and fragment ejection," International Journal of Impact Engineering, vol. 33, no. 1-12, pp. 703-712, 2006.

[10] M. Wicklein, S. Ryan, D. M. White, and R. A. Clegg, "Hypervelocity impact on CFRP: testing, material modelling, and numerical simulation," International Journal of Impact Engineering, vol. 35, no. 12, pp. 1861-1869, 2008.

[11] F. K. Schäfer, S. Ryan, M. Lambert, and R. Putzar, "Ballistic limit equation for equipment placed behind satellite structure walls," International Journal of Impact Engineering, vol. 35, no. 12, pp. 1784-1791, 2008.

[12] B. Jia, Z.-T. Ma, W. Zhang, and B.-J. Pang, "Numerical simulation investigation in ballistic limit of Whipple shield structure with Al-foam bumperb," Material Science and Technology, vol. 18, no. 3, pp. 368-372, 2010.

[13] Z. Zhang, R. Chi, B. Pang, and G. Guan, "A study on the split effect of projectile debris on honeycomb core," in Proceedings of the 4th International Conference on Sustainable Energy and Environmental Engineering, vol. 53, pp. 766-731, Shenzhen, China, December 2015.

[14] E. L. Christiansen, "Design and performance equations for advanced meteoroid and debris shields," International Journal of Impact Engineering, vol. 14, no. 1, pp. 145-156, 1993.

[15] W. P. Schonberg, F. Schäfer, and R. Putzar, "Predicting the perforation response of honeycomb sandwich panels using ballistic limit equations," Journal of Spacecraft and Rockets, vol. 46, no. 5, pp. 976-981, 2009. 


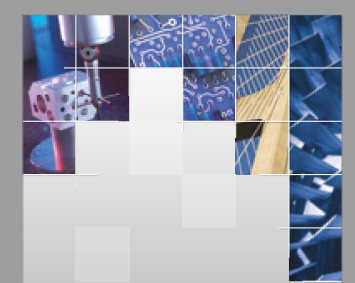

\section{Enfincering}
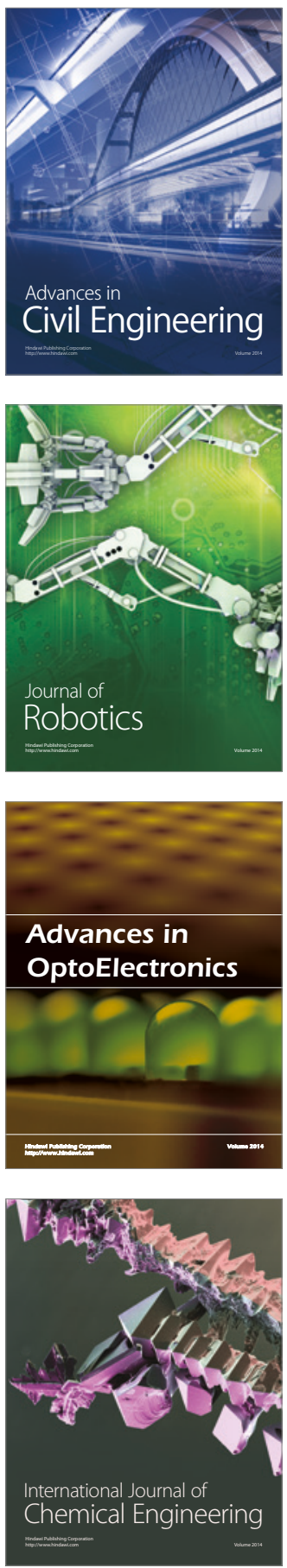

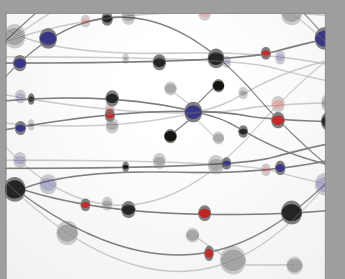

The Scientific World Journal

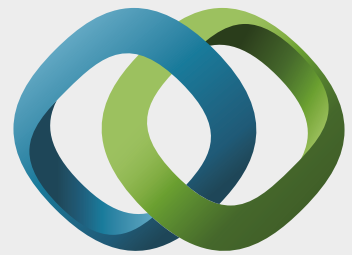

\section{Hindawi}

Submit your manuscripts at

https://www.hindawi.com
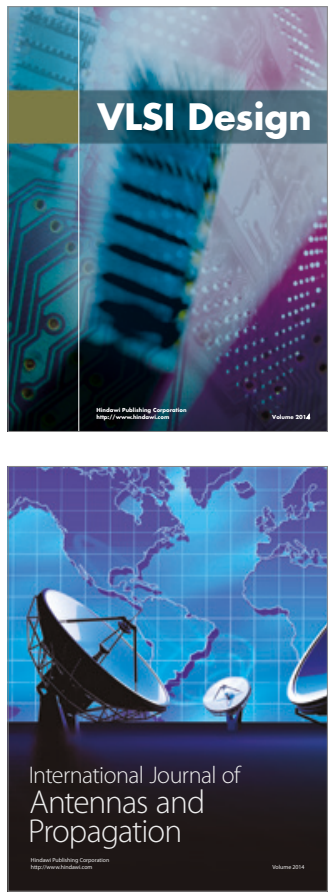

\section{Rotating}

Machinery
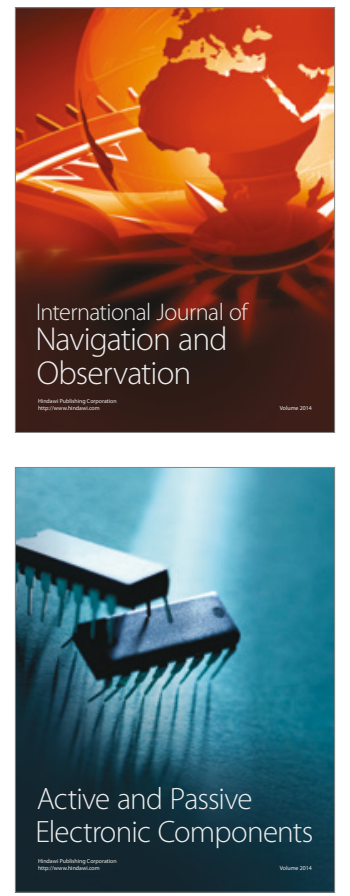
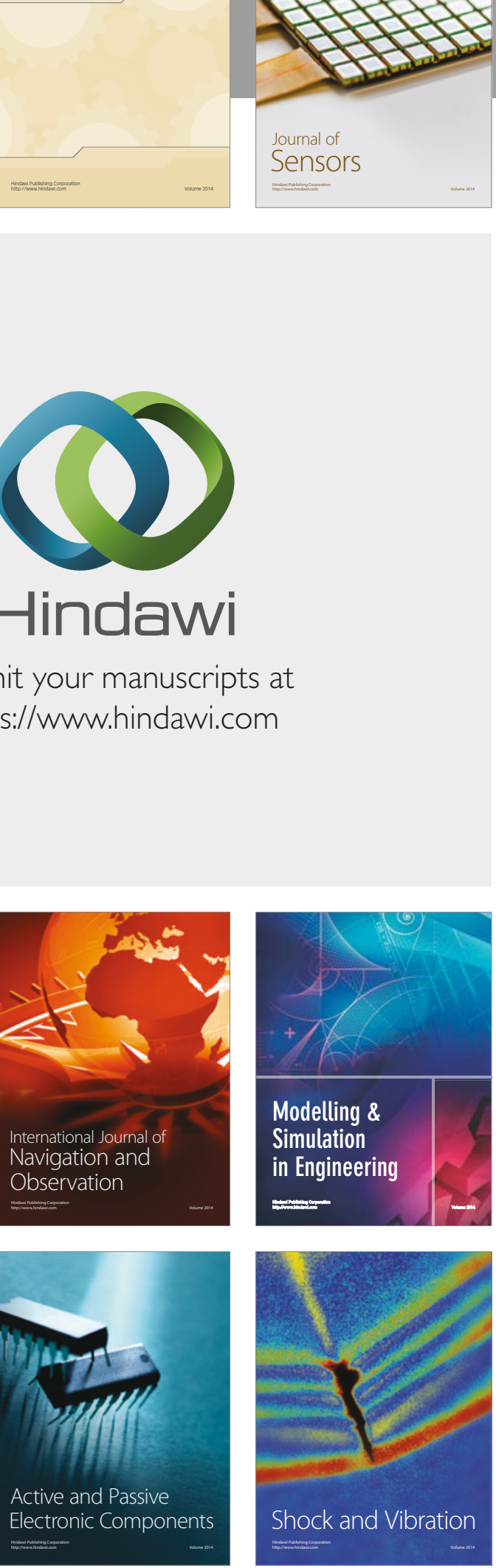
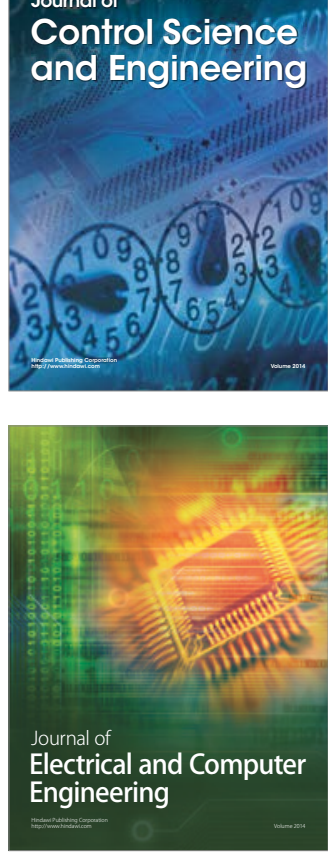

Distributed

Journal of

Control Science

and Engineering
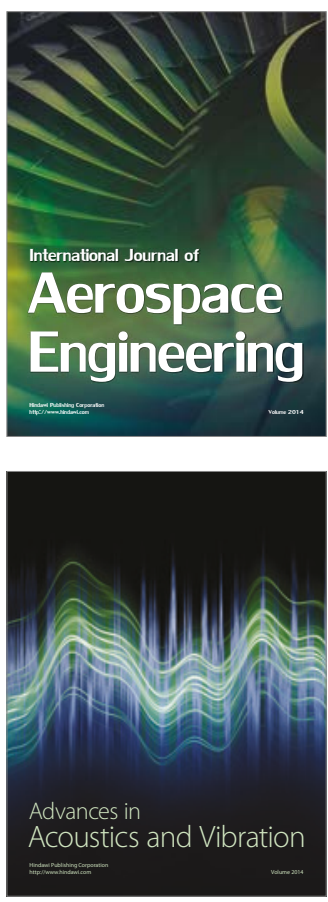

Sensor Networks 